\title{
Tolerance of Volume Control Noninvasive Ventilation in Subjects With Amyotrophic Lateral Sclerosis
}

\author{
Daniel Martínez MD, Jesús Sancho MD, Emilio Servera MD, and Julio Marín MD
}

\begin{abstract}
BACKGROUND: Noninvasive ventilation (NIV) tolerance has been identified as an independent predictor of survival in amyotrophic lateral sclerosis (ALS). Volume control continuous mandatory ventilation (VC-CMV) NIV has been associated with poor tolerance. The aim of this study was to determine the tolerance of subjects with ALS to VC-CMV NIV. METHODS: This was a prospective study involving subjects with ALS who were treated with VC-CMV NIV. Respiratory and functional parameters were recorded when the subjects began ventilatory support. NIV tolerance was evaluated after 3 months. RESULTS: Eighty-seven subjects with ALS were included. After 3 months, 80 subjects $(92 \%)$ remained tolerant of NIV. Tolerant subjects presented greater survival (median 22.0 months, 95\% CI 14.78-29.21) than intolerant subjects (median 6.0 months, 95\% CI 0.86-11.13 $)(P=.03)$. The variables that best predicted NIV tolerance were mechanically assisted cough peak flow $(P=.01)$ and percentage of time spent with $\mathrm{S}_{\mathrm{pO}_{2}}<90 \%$ at night while on NIV (P $=.03)$ CONCLUSIONS: VC-CMV NIV provides high rates of NIV tolerance in subjects with ALS. Mechanically assisted cough peak flow and percentage of time spent with $\mathrm{S}_{\mathrm{pO}_{2}}<90 \%$ at night while using NIV are the 2 factors associated with tolerance of VC-CMV NIV in subjects with ALS. Key words: noninvasive ventilation; respiratory failure; amyotrophic lateral sclerosis; neuromuscular disease; tolerance. [Respir Care 2015;60(12):1765-1771. (C) 2015 Daedalus Enterprises]
\end{abstract}

\section{Introduction}

Noninvasive ventilation (NIV) is recognized as being able to prolong survival, relieve symptoms, avoid hospitalizations, and improve quality of life in patients with

Dr Martínez is affiliated with the Respiratory Medicine Section, Hospital Marina Baixa, Villajoyosa, Alicante, Spain. Drs Sancho and Servera are affiliated with the Respiratory Care Unit, Respiratory Medicine Department, Hospital Clínico Universitario, and the Research Group for Respiratory Problems in Neuromuscular Disease, INCLIVA Institute of Health Research, Valencia, Spain. Dr Servera is affiliated with the Physical Therapy Department at Universitat de Valencia, Spain. Dr Marin is affiliated with the Medicine Department at Universitat de Valencia, Spain.

Supplementary material related to this paper is available at http:// www.rcjournal.com.

The authors have disclosed no conflicts of interest.

Correspondence: Jesús Sancho MD, Respiratory Care Unit, Respiratory Medicine Department, Hospital Clínico Universitario, Avenida Blasco Ibañez 17, 46010 Valencia, Spain. E-mail: jesus.sancho@uv.es.

DOI: $10.4187 /$ respcare.04172 amyotrophic lateral sclerosis (ALS).1,2 Survival of subjects with ALS has been found to be improved if NIV is used for at least 4 consecutive h during sleep ${ }^{3}$; thus, NIV adherence has been identified as an independent predictor of survival and quality of life benefit in patients with ALS. The identification of factors associated with NIV tolerance is a major issue. ${ }^{4}$ NIV is now delivered to patients with ALS mainly in either a pressure control (PC-CMV) or volume control (VC-CMV) continuous mandatory ventilation mode, but in the majority of studies that evaluated NIV tolerance in subjects with ALS, NIV was delivered by the PC-CMV mode.5,6 PC-CMV NIV was found to be associated with better tolerance in a previous study in which VC-CMV NIV was the most used mode in intolerant subjects with $\mathrm{ALS},{ }^{3}$ although no information about ventilator parameters and adverse effects related to each mode of ventilation was reported, and the choice of device was made by the subject after the 2 devices had been sampled. Coupled with its easier use, this has made the pressure-cycled mode the most frequently employed ventilator mode in ALS. ${ }^{7}$ However, it has recently been shown that, despite no differences in survival, VC-CMV NIV provides more effective ventilation than PC-CMV NIV in 
subjects with ALS, improving hypoventilation symptoms in a greater proportion of subjects with fewer nocturnal desaturations. ${ }^{8}$ The aim of this study was to determine the tolerance of subjects with ALS to VC-CMV NIV and whether a clinical or functional parameter can predict NIV tolerance in subjects with ALS treated with VC-CMV NIV.

\section{Methods}

We performed a prospective study from January 2006 to January 2014 at a respiratory care unit of a university hospital. We included all medically stable subjects with ALS for whom NIV was indicated in accordance with a modification of the criteria of the American College of Chest Physicians ${ }^{9}$ : presence of hypoventilation symptoms or at least one physiological criterion such as an FVC of $<50 \%$ of predicted, a maximum inspiratory pressure $\left(\mathrm{P}_{\text {Imax }}\right)$ of $<60 \%$ of predicted, a nocturnal $\mathrm{S}_{\mathrm{pO}_{2}}$ of $<90 \%$ during $>5 \%$ of an overnight recording, and a morning $\mathrm{P}_{\mathrm{aCO}_{2}}$ of $>45 \mathrm{~mm} \mathrm{Hg}$. The protocol was approved by the hospital's ethics committee, and informed consent was obtained from each subject who took part in the study. Exclusion criteria were: refusal to participate in the study, presence of previous chronic pulmonary or airway disease, substance abuse, another associated rapidly progressing disease with a survival expectancy of $<1$ month, severe frontotemporal dementia that might compromise NIV tolerance, and refusal of NIV.

\section{Clinical and Functional Impairment Assessment}

Subjects were diagnosed as having definite or probable ALS in accordance with the El Escorial World Federation of Neurology revised criteria. ${ }^{10}$ Neurological and respiratory functions were assessed regularly, and demographic data were collected upon NIV indication. Demographic data included sex, age, body mass index, and the site of ALS onset. Functional impairment was evaluated using the revised Amyotrophic Lateral Sclerosis Functional Rating Scale (ALSFRS-R), ${ }^{11}$ and bulbar involvement was assessed in accordance with the Norris scale bulbar subscore. ${ }^{12}$ (See the supplementary materials at http:// www.rcjournal.com for a description of the Norris scale bulbar subscore.) All subjects received therapeutic procedures (multidisciplinary care, scheduled clinical assessments, nutritional support, psychological management, neurological treatment, and sialorrhoea treatment) in accordance with expert guidelines. ${ }^{13}$

Upon NIV initiation, spirometry was assessed with a pneumotachograph spirometer (MS 2000, C Schatzman, Madrid, Spain) in accordance with European Respiratory Society guidelines and suggested values, ${ }^{14}$ and $\mathrm{P}_{\mathrm{Imax}}$ and maximum expiratory pressure were measured (Electrometer 78.905a, Hewlett-Packard, Palo Alto, California) ac-

\section{QUICK LOOK}

\section{Current knowledge}

Noninvasive ventilation (NIV) prolongs survival, relieves symptoms, avoids hospitalizations, and improves quality of life in patients with amyotrophic lateral sclerosis (ALS). Survival has been found to be improved in subjects with ALS if NIV is used for $\geq 4$ consecutive $h$ during sleep. NIV adherence has been identified as an independent predictor of survival and quality of life in patients with ALS.

\section{What this paper contributes to our knowledge}

Ninety-two percent of subjects with ALS using volume control NIV for 3 months remained tolerant. Tolerant subjects presented greater survival than intolerant subjects. The variables that best predicted NIV tolerance were mechanically assisted cough peak flow and percentage of time spent with $\mathrm{S}_{\mathrm{pO}_{2}}<90 \%$ at night while on NIV. Volume control NIV in the continuous mandatory ventilation mode was well tolerated in this group of subjects with ALS.

cording to the Black and Hyatt technique. ${ }^{15}$ Cough peak flow, maximum insufflation capacity, and manually and mechanically assisted cough peak flows were assessed with a sealed oronasal mask (King Systems, Noblesville, Indiana) and a pneumotachograph spirometer (MS 2000) as described previously. ${ }^{16}$ Arterial blood gas analysis were also performed (ABL 500, Radiometer, Brønshøj, Denmark).

Overnight pulse oximetry readings were taken in the hospital during continuous nocturnal cardiorespiratory monitoring (MEC-1000, Shenzhen Mindray Biomedical Electronics, Shenzhen, China). The percentage of time spent with $\mathrm{S}_{\mathrm{pO}_{2}}<90 \%$ at night, the minimum nocturnal $\mathrm{S}_{\mathrm{pO}_{2}}$, and the mean nocturnal $\mathrm{S}_{\mathrm{pO}_{2}}$ were recorded while the subject was spontaneously breathing.

NIV was delivered via a portable ventilator in the VCCMV mode (PV 501 and PV 403, Breas Medical, Mölnlycke, Sweden; AiroxHome2 and Legendair, Airox, Pau, France). Ventilator adjustments were performed in the hospital during nocturnal cardiorespiratory monitoring. NIV was delivered through oronasal masks (Mirage, ResMed, Madrid, Spain), lip-seal mouthpiece (Tyco-Puritan Bennett, Pleasanton, California), or nasal interfaces (Healthdyne, Marietta, Georgia) during the night to optimize comfort and minimize air leaks. The ventilator was initially adjusted to obtain a tidal volume of $\sim 10 \mathrm{~mL} / \mathrm{kg}$, an inspiratory-expiratory ratio of 1:1.2 or 1:1.5, a backup breathing frequency near that of spontaneous breathing, and an inspiratory trigger sensitivity of $-0.5 \mathrm{~cm} \mathrm{H}_{2} \mathrm{O}$. The ven- 
tilator settings were then readjusted during the night based on the subjects' comfort levels to achieve effective ventilation. Ventilation was considered to be effective when the percentage of time spent with $\mathrm{S}_{\mathrm{pO}_{2}}<90 \%$ at night while on NIV was $<5 \%$, the $\mathrm{P}_{\mathrm{aCO}}$ while on NIV was $<45 \mathrm{~mm}$ $\mathrm{Hg}$, and hypoventilation symptoms were avoided. ${ }^{17} \mathrm{Hy}-$ poventilation symptoms included orthopnea, dyspnea, poor sleep, excessive daytime sleepiness, poor concentration, and morning headache. ${ }^{9,13}$ Before discharge from the hospital, when effective ventilation (or if this was not possible, an improved ventilatory situation) had been achieved, overnight pulse oximetry and gas exchange parameters with NIV were recorded for analysis as part of the research protocol.

In those subjects in whom, despite effective nocturnal NIV, symptoms of hypoventilation, hypercapnia, or respiratory accessory muscle use persisted, daytime NIV was adjusted through a mouthpiece, lip-seal mouthpiece, or nasal pillow interfaces, as needed. In those subjects with cough peak flow levels $<4.25 \mathrm{~L} / \mathrm{s}$, mechanically assisted coughing was prescribed. ${ }^{16}$

After starting home NIV, a clinical and functional assessment was scheduled every 3 months. Subjects were defined as tolerant of NIV if they could use the ventilator nightly for $>4$ consecutive $\mathrm{h}^{3}$ as recorded by the machine counter and according to information provided by each subject. Survival constituted the period from NIV initiation to tracheostomy or death if the subject refused a tracheostomy. Tracheostomy was considered if NIV could not provide adequate alveolar ventilation, when mechanically assisted coughing was inadequate to remove airway secretions causing an $\mathrm{S}_{\mathrm{pO}_{2}}$ of $<95 \%$, and/or subjects felt continuously encumbered or dyspneic.

\section{Statistical Analysis}

Continuous normally distributed variables were expressed as mean $\pm \mathrm{SD}$. Binary and categorical variables were summarized using frequency counts and percentages. Data comparisons were performed using the Student paired $t$ test, and categorical variables were compared with the chi-square test. When the variables did not have a normal distribution, the Mann-Whitney test and Wilcoxon test for paired data were used. Comparisons between assisted and unassisted cough peak flows were made with an analysis of variance for repeated measurements. Univariate and multivariate logistic regression analyses were used to determine those variables that were independently associated with NIV tolerance. Long-term survival was assessed with Kaplan-Meier charts, applying the log-rank test to compare differences in survival between tolerant and intolerant subjects. Statistical significance was taken as $P<.05$.

\section{Results}

During the study period, 105 subjects with ALS were considered eligible for NIV. Eighteen patients were excluded: 14 directly declined NIV treatment, 3 suffered from severe frontotemporal dementia, and one presented with concomitant bronchial pathology. Overall, 87 subjects were prescribed VC-CMV NIV. At the time of NIV initiation, 58 subjects $(66.6 \%)$ were using mechanically assisted coughing, and 31 subjects (35.6\%) had a percutaneous endoscopic gastrostomy for enteral nutrition. Time from ALS onset to NIV initiation was $30.4 \pm 37.4$ months (median 23.5 months). Data on demographics, respiratory function, and functional assessment of the subjects included in the study upon NIV initiation are provided in Table 1. Differences between tolerant and intolerant subjects were found in mechanically assisted cough peak flow; statistical differences were found between cough peak flow, manually assisted cough peak flow, and mechanically assisted cough peak flow $(P<.001)$ in tolerant subjects but not in intolerant subjects $(P=.29)$

NIV improved nocturnal oximetry features and gas exchange parameters (Table 2). The mean tidal volume used was $778 \pm 131 \mathrm{~mL}$, the backup breathing frequency was $14 \pm 1$ breaths $/ \mathrm{min}$, and the most frequent interface used at night was an oronasal mask (88.5\%). The mean length of hospital stay for NIV adjustment was $4.7 \pm 1.8 \mathrm{~d}$. After ventilator adjustment and at the time of home discharge, all subjects were tolerant to NIV, but 3 months after NIV adaptation, 7 subjects ( $8 \%$ ) were found to be intolerant of NIV. The mean hours/d of NIV use reported at 3 months after NIV adaptation were $9.8 \pm 4.5$ in the tolerant group and $1.4 \pm 0.5$ in the intolerant group. Statistical differences were found between the tolerant and intolerant subjects only in mechanically assisted cough peak flow (see Table 1). No statistical differences were found between tolerant and intolerant subjects in baseline blood gas and nocturnal gas exchange parameters. At the time of NIV indication, 67 subjects (77\%) presented symptoms related to hypoventilation (76.2\% in the tolerant group and $85.7 \%$ in the intolerant group, $P=.32$ ); hypoventilation symptoms persisted at 3 months in 6 tolerant subjects $(7.5 \%)$ and in 2 intolerant subjects $(71.4 \%)(P<.01)$. During NIV adaptation, effective ventilation was achieved in $81.2 \%$ of the tolerant group and in $85.7 \%$ of the intolerant group $(P=.40)$.

After the scheduled clinical and functional visit at 3 months, those subjects identified as intolerant were hospitalized to improve NIV adherence. Overnight continuous cardiorespiratory monitoring while on NIV was performed, and no statistical differences were found in the nocturnal oximetry data, gas exchange parameters, and ventilation effectiveness recorded at NIV initiation and after 3 months (percentage of time spent with $\mathrm{S}_{\mathrm{pO}_{2}}<90 \%$ at night while on NIV: $6 \pm 8$ vs $5 \pm 7 \%, P=.13$; minimum nocturnal 
Table 1. Baseline Demographic and Pulmonary Function Parameters for Tolerant and Intolerant Subjects With ALS

\begin{tabular}{|c|c|c|c|}
\hline Parameter & Tolerant $(n=80)$ & Intolerant $(n=7)$ & $P$ \\
\hline Sex (males/females), $n$ & $40 / 40$ & $2 / 5$ & .27 \\
\hline Age, mean \pm SD y & $62.9 \pm 9.0$ & $64.1 \pm 8.0$ & .70 \\
\hline $\mathrm{BMI}$, mean $\pm \mathrm{SD} \mathrm{kg} / \mathrm{m}^{2}$ & $26.3 \pm 4.8$ & $26.8 \pm 3.0$ & .68 \\
\hline Onset (spinal/bulbar), $n$ & $56 / 24$ & $5 / 2$ & .93 \\
\hline Time from ALS onset to NIV, mean $\pm \mathrm{SD}$ mo & $20.0 \pm 14.6$ & $33.5 \pm 30.8$ & .21 \\
\hline ALSFRS-R, mean \pm SD & $30.4 \pm 7.1$ & $28.3 \pm 3.7$ & .26 \\
\hline Norris scale bulbar subscore, mean $\pm \mathrm{SD}$ & $29.3 \pm 9.2$ & $24.1 \pm 8.1$ & .15 \\
\hline $\mathrm{FVC}$, mean $\pm \mathrm{SD} \mathrm{L}$ & $1.61 \pm 1.0$ & $1.23 \pm 0.5$ & .09 \\
\hline $\mathrm{FVC}$, mean $\pm \mathrm{SD} \%$ predicted & $52.8 \pm 24.3$ & $49.2 \pm 18.4$ & .63 \\
\hline Maximum insufflation capacity, mean \pm SD L & $2.2 \pm 1.0$ & $1.4 \pm 0.4$ & .12 \\
\hline Cough peak flow, mean \pm SD L/s & $3.8 \pm 1.9$ & $2.9 \pm 1.7$ & .07 \\
\hline Manually assisted cough peak flow, mean \pm SD L/s & $4.6 \pm 2.1$ & $3.0 \pm 1.2$ & .10 \\
\hline Mechanically assisted cough peak flow, mean $\pm \mathrm{SD}$ L/s & $4.1 \pm 1.1$ & $2.9 \pm 0.7$ & .01 \\
\hline $\mathrm{P}_{\text {Imax }}$, mean $\pm \mathrm{SD} \mathrm{cm} \mathrm{H}_{2} \mathrm{O}$ & $-48.0 \pm 23.5$ & $-38.3 \pm 24.2$ & .38 \\
\hline $\mathrm{P}_{\mathrm{Emax}}$, mean $\pm \mathrm{SD} \mathrm{cm} \mathrm{H} \mathrm{H}_{2} \mathrm{O}$ & $74.3 \pm 42.9$ & $60.2 \pm 51.7$ & .54 \\
\hline $\begin{array}{l}\text { ALS }=\text { amyotrophic lateral sclerosis } \\
\text { BMI }=\text { body mass index } \\
\text { NIV = noninvasive ventilation } \\
\text { ALSFRS- } R \text { = revised Amyotrophic Lateral Sclerosis Functional Rating Scale } \\
P_{\text {Imax }}=\text { maximum inspiratory pressure } \\
P_{\text {Emax }}=\text { maximum expiratory pressure }\end{array}$ & & & \\
\hline
\end{tabular}

Table 2. Blood Gas and Nocturnal Gas Exchange Parameters at Baseline and After NIV Initiation

\begin{tabular}{|c|c|c|c|c|c|c|c|}
\hline \multirow{2}{*}{ Parameter } & \multicolumn{3}{|c|}{ Tolerant } & \multicolumn{3}{|c|}{ Intolerant } & \multirow{2}{*}{$\begin{array}{c}P(\text { Tolerant vs } \\
\text { Intolerant, Using NIV) }\end{array}$} \\
\hline & Baseline & NIV & $P$ & Baseline & NIV & $P$ & \\
\hline $\mathrm{pH}$ & $7.39 \pm 0.03$ & $7.42 \pm 0.03$ & .01 & $7.41 \pm 0.03$ & $7.40 \pm 0.01$ & .46 & .003 \\
\hline $\mathrm{P}_{\mathrm{aO}_{2}}, \mathrm{~mm} \mathrm{Hg}$ & $75 \pm 12$ & $84 \pm 10$ & .01 & $75 \pm 2$ & $76 \pm 3$ & .41 & .01 \\
\hline $\mathrm{P}_{\mathrm{aCO}_{2}}, \mathrm{~mm} \mathrm{Hg}$ & $50 \pm 8$ & $42 \pm 4$ & .01 & $44 \pm 7$ & $44 \pm 3$ & .94 & .24 \\
\hline $\mathrm{HCO}_{3}{ }^{-}, \mathrm{mmol} / \mathrm{L}$ & $28 \pm 2$ & $26 \pm 2$ & .01 & $27 \pm 2$ & $26 \pm 2$ & .038 & .94 \\
\hline $\mathrm{S}_{\mathrm{aO}_{2}}, \%$ & $93 \pm 2$ & $96 \pm 1$ & .01 & $93 \pm 1$ & $94 \pm 1$ & .18 & .01 \\
\hline $\begin{array}{l}\text { Time spent with } \mathrm{S}_{\mathrm{pO}_{2}}<90 \% \text { at } \\
\text { night while on NIV, } \%\end{array}$ & $33 \pm 29$ & $1 \pm 3$ & .01 & $21 \pm 28$ & $6 \pm 8$ & .24 & .01 \\
\hline Minimum nocturnal $\mathrm{S}_{\mathrm{pO}_{2}}, \%$ & $77 \pm 10$ & $92 \pm 3$ & .01 & $83 \pm 6$ & $89 \pm 1$ & .042 & .01 \\
\hline Mean nocturnal $\mathrm{S}_{\mathrm{pO}_{2}}, \%$ & $91 \pm 3$ & $95 \pm 1$ & .01 & $91 \pm 2$ & $94 \pm 1$ & .01 & .01 \\
\hline
\end{tabular}

$\mathrm{S}_{\mathrm{pO}_{2}}: 89 \pm 1$ vs $89 \pm 2 \%, P=.51$; mean nocturnal $\mathrm{S}_{\mathrm{pO}_{2}}$ : $94 \pm 1 \mathrm{vs} 94 \pm 1 \%, P=.23 ; \mathrm{P}_{\mathrm{aCO}_{2}}: 44 \pm 3 \mathrm{vs} 43 \pm 3 \mathrm{~mm}$ $\mathrm{Hg}, P=.26$; effective NIV: 85.71 vs $85.71 \%$ ). The causes for poor tolerance reported by subjects were: problems related to the interface in one subject, refusal of NIV treatment by 3 subjects, and episodes of sudden breathlessness during NIV in 3 subjects. During the nocturnal monitoring, it was found that these breathless episodes were preceded by a reduction or abolition of respiratory thoracoabdominal movements, an increase in peak inspiratory pressure, and air leaks around the mask with no evidence of retained secretions. For all intolerant subjects, despite changes in masks and ventilator parameters and even trans- fer to PC-CMV NIV, the situation regarding NIV tolerance did not improve.

Table 3 shows the results of the univariate logistic regression analysis that was carried out to establish predictive factors of NIV tolerance in subjects with ALS. In the multivariate logistic regression analysis, the only variables that predicted NIV tolerance in ALS were mechanically assisted cough peak flow (odds ratio 6.80, 95\% CI 1.41$32.63, P=.01$ ) and percentage of time spent with $\mathrm{S}_{\mathrm{pO}_{2}}<$ $90 \%$ at night while on NIV (odds ratio $0.81,95 \% \mathrm{CI}$ 0.67-0.98, $P=.037$ ).

No subject died during the first 3 months of the study. The mean time from NIV initiation to death/tracheostomy 
Table 3. Predictors of NIV Tolerance in Subjects With ALS: Univariate Analysis

\begin{tabular}{|c|c|c|}
\hline Predictor & OR $(95 \% \mathrm{CI})$ & $P$ \\
\hline Age & $0.98(0.09-1.07)$ & .71 \\
\hline Sex & $2.50(0.45-13.64)$ & .29 \\
\hline BMI & $0.97(0.83-1.14)$ & .76 \\
\hline ALS onset & $0.93(0.16-5.15)$ & .93 \\
\hline ALSFRS-R & $1.04(0.92-1.17)$ & .47 \\
\hline Norris scale bulbar subscore & $1.06(0.97-1.15)$ & .16 \\
\hline FVC & $2.01(0.56-7.19$ & .27 \\
\hline$\%$-predicted FVC & $1.01(0.97-1.04)$ & .69 \\
\hline $\mathrm{P}_{\operatorname{Imax}}$ & $0.97(0.92-1.02)$ & .33 \\
\hline $\mathrm{P}_{\text {Emax }}$ & $1.01(0.98-1.03)$ & .44 \\
\hline Cough peak flow & $1.49(0.78-2.84)$ & .21 \\
\hline Maximum insufflation capacity & $4.10(0.75-22.37)$ & .10 \\
\hline Manually assisted cough peak flow & $1.91(0.86-4.20)$ & .10 \\
\hline $\begin{array}{l}\text { Mechanically assisted cough } \\
\text { peak flow }\end{array}$ & $6.64(1.61-27.32)$ & .01 \\
\hline Hypoventilation symptoms & $1.86(0.21-16.51)$ & .57 \\
\hline $\mathrm{P}_{\mathrm{aO}_{2}}$ & $0.98(0.93-1.06)$ & .95 \\
\hline $\mathrm{P}_{\mathrm{aCO}_{2}}$ & $1.11(0.98-1.26)$ & .07 \\
\hline $\begin{array}{l}\% \text { Time spent with } \mathrm{S}_{\mathrm{pO}_{2}}<90 \% \\
\text { at night while on NIV }\end{array}$ & $1.01(0.98-1.01)$ & .30 \\
\hline Minimum nocturnal $\mathrm{S}_{\mathrm{pO}_{2}}$ & $0.91(0.79-1.04)$ & .18 \\
\hline Mean nocturnal $\mathrm{S}_{\mathrm{pO}_{2}}$ & $1.01(0.78-1.28)$ & .96 \\
\hline $\mathrm{P}_{\mathrm{aCO}_{2}}-\mathrm{NIV}$ & $0.90(0.75-1.07)$ & .23 \\
\hline $\mathrm{P}_{\mathrm{aO}_{2}}-\mathrm{NIV}$ & $1.10(0.99-1.22)$ & .054 \\
\hline $\begin{array}{l}\% \text { Time spent with } \mathrm{S}_{\mathrm{pO}_{2}}<90 \% \\
\text { at night-NIV }\end{array}$ & $0.82(0.71-0.95)$ & .01 \\
\hline Minimum nocturnal $\mathrm{S}_{\mathrm{pO}_{2}}-\mathrm{NIV}$ & $1.31(1.06-1.62)$ & .01 \\
\hline Mean nocturnal $\mathrm{S}_{\mathrm{pO}_{2}}-\mathrm{NIV}$ & $1.56(1.02-2.38)$ & .039 \\
\hline Effective NIV & $2.06(0.36-11.79)$ & .41 \\
\hline $\begin{array}{l}\text { NIV }=\text { noninvasive ventilation } \\
\text { ALS }=\text { amyotrophic lateral sclerosis } \\
\text { OR }=\text { odds ratio } \\
\text { BMI = body mass index } \\
\text { ALSFRS-R = revised Amyotrophic Lateral Scle } \\
\text { PImax }=\text { maximum inspiratory pressure }_{\text {I }_{\text {Emax }} \text { maximum expiratory pressure }}\end{array}$ & unctional Rating Scale & \\
\hline
\end{tabular}

in tolerant subjects was $44.9 \pm 7.6$ months (median 22.0 months, 95\% CI 14.78-29.21); the mean time from NIV initiation to death/tracheostomy in intolerant subjects was $14.4 \pm 5.2$ months (median 6.0 months, 95\% CI $0.86-$ 11.13). Statistical differences were found in time spent after NIV initiation between the 2 groups of subjects $(P=$ .030) (Fig. 1)

\section{Discussion}

This study shows that in those subjects with ALS using home VC-CMV NIV, the rate of tolerance 3 months after NIV initiation was high. This finding clearly improves current knowledge about the usefulness of VC-CMV NIV in patients with ALS. Furthermore, those subjects with

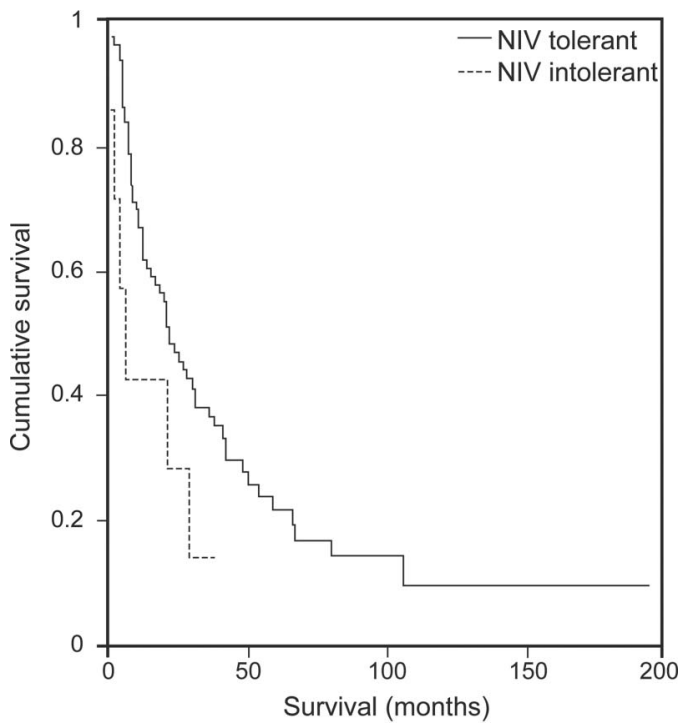

Fig. 1. Survival without tracheostomy from initiation of noninvasive ventilation (NIV).

ALS who presented a lower cough peak flow generated with mechanically assisted coughing and more time spent with $\mathrm{S}_{\mathrm{pO}_{2}}<90 \%$ during NIV at night were predisposed to have low adherence to NIV. However, we are currently unable to provide a convincing explanation for this latter phenomenon.

Most of the studies focusing on NIV tolerance in subjects with ALS have been conducted using PC-CMV NIV, and the reported tolerance levels range from 46 to $90 \% .^{3-6,18}$ Although Aboussouan et $\mathrm{al}^{3}$ found that NIV tolerance in subjects with ALS was higher with PC-CMV, the findings of the present study, using only VC-CMV NIV, show a rate of tolerance of $92 \%$, similar to the best results in the literature. In previous studies focusing on a heterogeneous population including neuromuscular subjects, VC-CMV NIV was associated with more gastrointestinal adverse effects, ${ }^{19,20}$ mainly flatulence, although no differences between the 2 ventilator modes in overall acceptability have been reported. ${ }^{20,21}$ Our results show high rates of tolerance, even higher than those reported in studies using PC-CMV NIV, and point to an important role for this ventilator mode when it is initiated in a specific unit for the management of respiratory problems in patients with ALS. The mean set tidal volume used in the present study is in the range of those described in previous studies, and no adverse effects have been reported. ${ }^{1,8,21}$

Survival of subjects with ALS has been shown to be strongly associated with NIV adherence, ${ }^{3,22}$ and NIV adherence is a predictor of the improvement produced by NIV in quality of life in subjects with ALS. ${ }^{4}$ Our results confirm, in accordance with previous studies, that tolerant subjects use NIV for a longer period before death/tracheostomy (median 22.0 months) compared with intolerant 
subjects (median 6.0 months). In a recent collaboration, ${ }^{8}$ we showed that both ventilator modes provided similar results for survival in subjects with ALS, but VC-CMV NIV seemed to provide more effective ventilation, with less time spent with $\mathrm{S}_{\mathrm{pO}_{2}}<90 \%$ at night and higher minimum nocturnal $\mathrm{S}_{\mathrm{pO}_{2}}$ and mean $\mathrm{S}_{\mathrm{pO}_{2}}$ while on NIV. If our results are compared with those from another study using PC-CMV NIV, ${ }^{6}$ the nocturnal oxygenation of our tolerant group is largely greater. Moreover, we found that VCCMV NIV was more effective in relieving symptoms of hypoventilation and was associated with less frequent changes to ventilator parameters over time. ${ }^{8}$

This study shows that NIV tolerance was associated with time spent with $\mathrm{S}_{\mathrm{pO}_{2}}<90 \%$ at night while on NIV. Although differences between tolerant and intolerant subjects were not found in the percentage of subjects in whom effective NIV (as defined in methods section) was achieved, for those subjects with low NIV adherence, no improvement in $\mathrm{P}_{\mathrm{aO}}$ and $\mathrm{P}_{\mathrm{aCO}}$ was found, and they presented lower $\mathrm{P}_{\mathrm{aO}_{2}}$ after NIV and worse minimum nocturnal $\mathrm{S}_{\mathrm{pO}_{2}}$ and mean nocturnal $\mathrm{S}_{\mathrm{pO}_{2}}$ while using NIV compared with tolerant subjects. In the same way, the percentage of effective NIV in intolerant subjects was the same upon NIV initiation and after 3 months $(85.71 \%)$; this means that there was no worsening in the effectiveness of ventilation during this 3-month period to which intolerance might otherwise be attributed. In 3 of the intolerant subjects, the desaturation episodes were preceded by a reduction or abolition of respiratory thoracoabdominal movements, an increase in peak inspiratory pressure, and air leaks around the mask without evidence of retained secretions. This is suggestive of central breathing pause despite the use of NIV, which has been described as a cause of patientventilator asynchrony in subjects with ALS, ${ }^{23}$ mainly when there is bulbar impairment. Patient-ventilator asynchrony can lead to a decrease in NIV adherence. ${ }^{23-25}$ In our subjects, these episodes and low NIV adherence persisted despite a transfer to PC-CMV NIV, with this falling outside of the study protocol.

Severe bulbar dysfunction is a major factor associated with poor NIV tolerance and can impair the effectiveness of NIV in subjects with ALS, 3,4,6,26 whereas the presence of hypoventilation symptoms upon NIV initiation, mainly orthopnea or dyspnea, has been identified as a predictor of good adherence. ${ }^{4,27}$ The results of our study did not demonstrate a relationship between bulbar dysfunction as measured by the Norris scale bulbar subscore or the site of ALS onset and NIV tolerance. There is no accepted standard with which to measure bulbar involvement, and most of the studies that have reported bulbar dysfunction as a predictor of NIV tolerance were conducted using a subjective assessment of bulbar function. ${ }^{3,26}$ In the study by Vandenberghe et al, ${ }^{6}$ the Norris scale bulbar subscores of tolerant subjects $(30.5 \pm 8.9)$ were similar to those of our tolerant subjects $(29.2 \pm 9.2)$, and there were statistical differences from the subscores of intolerant subjects (22.3 \pm $12.8)$, which were slightly lower than ours $(24.1 \pm 8.0)$. In fact, in both studies, the functional (ALSFRS-R) and respiratory (FVC) impairment of the included subjects is similar. Vandenberghe et $\mathrm{al}^{6}$ found that normal bulbar function upon NIV initiation, advanced age, and absence of accumulated airway secretions were the predictive factors associated with good NIV tolerance in subjects with ALS. Bulbar dysfunction is associated with more difficult secretion management and drooling, ${ }^{1}$ which could interfere with the effectiveness of and adherence to NIV treatment; thus, we always pay special attention to controlling the problems related to bulbar dysfunction that could affect NIV adherence. It is therefore surprising that, with no clinical or biological evidence that respiratory secretions interfered in the management of our intolerant subjects, mechanically assisted cough peak flow was a predictive factor of nonadherence. It may be that the fact that differences were found between cough peak flow, manually assisted cough peak flow, and mechanically assisted cough peak flow in the tolerant group but not in the intolerant group is important; exactly how important is unclear. The lack of an increase in cough peak flow when assisted coughing techniques have been applied has been proposed as a sign of bulbar dysfunction by Bach et $\mathrm{al},{ }^{28}$ so our findings could be an indirect expression of greater severity of bulbar impairment in our intolerant subjects. Thus, a more accurate tool to detect and quantify the severity of bulbar dysfunction would be desirable to explain some of the findings of the present study.

The limitations of this study include the lack of a control group and the small number of intolerant subjects. We have used VC-CMV with our patients for a number of years. We have previously studied various aspects of this procedure, ${ }^{29}$ and we have compared our results with those of a group with expertise in PC-CMV who take a similar approach to ours with regard to respiratory secretion management. ${ }^{8}$ From an ethical standpoint, the procedure we use with our patients must be the one in which we have the most expertise; therefore, we acknowledge possible limitations in our method, most notably the lack of a control group. Taking an honest and realistic view, most of our subjects would not have understood why we were presenting them with a situation in which randomization would have been required: that the treatment they were to receive would be decided by chance, rather than one proposed by their doctor as the best option for them. It is our opinion that ethical considerations mean that it is not always possible to employ the best methodological practices from a scientific point of view when carrying out clinical research on subjects with ALS..$^{30}$ In this case, an important negative effect of the absence of a randomized control group is the small number of subjects presenting with poor tolerance, 


\section{NIV Tolerance in Subjects With ALS}

and the large difference between the number of tolerant and intolerant subjects undermines the reliability of our results.

\section{Conclusions}

In summary, VC-CMV NIV provides high rates of NIV tolerance in subjects with ALS. Furthermore, although we are unable to provide convincing arguments to explain why, mechanically assisted cough peak flow and percentage of time spent with $\mathrm{S}_{\mathrm{pO}_{2}}<90 \%$ at night while on NIV are the 2 factors associated with tolerance of VC-CMV NIV in subjects with ALS.

\section{REFERENCES}

1. Bach JR. Amyotrophic lateral sclerosis: prolongation of life by noninvasive respiratory aids. Chest 2002;122(1):92-98.

2. Bourke SC, Tomlinson M, Williams TL, Bullock RE, Shaw PJ, Gibson GJ. Effects of non-invasive ventilation on survival and quality of life in patients with amyotrophic lateral sclerosis: a randomised controlled trial. Lancet Neurol 2006;5(2):140-147.

3. Aboussouan LS, Khan SU, Meeker DP, Stelmach K, Mitsumoto H. Effect of noninvasive positive-pressure ventilation on survival in amyotrophic lateral sclerosis. Ann Intern Med 1997;127(6):450-453.

4. Bourke SC, Bullock RE, Williams TL, Shaw PJ, Gibson GJ. Noninvasive ventilation in ALS. Indications and effect on quality of life. Neurology 2003;61(2):171-177.

5. Lo Coco D, Marchese S, Pesco MC, La Bella V, Piccoli F, Lo Coco A. Noninvasive positive-pressure ventilation in ALS. Predictors of tolerance and survival. Neurology 2006;67(5):761-765.

6. Vandenberghe N, Vallet AE, Petitjean T, Le Cam P, Peysson S, Guérin $\mathrm{C}$, et al. Absence of airway secretion accumulation predicts tolerance to noninvasive ventilation in ALS. Respir Care 2013;58(9): 1424-1432.

7. Radunovic A, Annane D, Jewitt K, Mustfa N. Mechanical ventilation for amyotrophic lateral sclerosis/motor neuron disease. Cochrane Database Syst Rev 2009;(4):CD004427.

8. Sancho J, Servera E, Morelot-Panzini C, Salachas F, Similowski T, Gonzalez-Bermejo J. Noninvasive ventilation effectiveness and the effect of ventilatory mode on survival in ALS patients. Amyotroph Lateral Scler Frontotemporal Degener 2014;15(1-2):55-61.

9. Consensus Conference Report. Clinical indications for noninvasive positive pressure ventilation in chronic respiratory failure due to restrictive lung disease, COPD, and nocturnal hypoventilation. Chest 1999;116(2):521-534

10. Brooks BR, Miller RG, Swash M, Munsat TL, World Federation of Neurology Research Group on Motor Neuron Diseases. El Escorial revisited: revised criteria for the diagnosis of amyotrophic lateral sclerosis. Amyotroph Lateral Scler Other Motor Neuron Disord 2000; 1(5):293-299.

11. Cedarbaum JM, Stambler N, Malta E, Fuller C, Hilt D, Thurmond B, Nakanishi A. The ALSFRS-R: a revised ALS functional rating scale that incorporates assessments of respiratory function. BDNF ALS Study Group (Phase III). J Neurol Sci 1999;169(1-2):13-21.

12. Lacomblez L, Bouche P, Bensimon G, Meininger V. A double-blind, placebo-controlled trial of high doses of gangliosides in amyotrophic lateral sclerosis. Neurology 1989;39(12):1635-1637.

13. Miller RG, Jackson CE, Kasarskis EJ, England JD, Forshew D, Johnston W, et al. Practice parameter update: the care of the patient with amyotrophic lateral sclerosis: Drug, nutritional, and respiratory therapies (an evidence-based review): report of the Quality Standards Subcommittee of the American Academy of Neurology. Neurology 2009;73(15):1218-1226.

14. Quanjer PH, Tammeling GJ, Cotes JE, Pedersen OF, Peslin R, Yernault JC. Lung volumes and forced ventilatory flows. Report of Working Party Standardization of Lung Function Tests, European Community for Steel and Coal. Official Statement of the European Respiratory Society. Eur Respir J Suppl 1993;16:5-40.

15. Black LF, Hyatt RE. Maximal respiratory pressures: normal values and relationship to age and sex. Am Rev Respir Dis 1969;99(5):696702 .

16. Sancho J, Servera E, Díaz J, Marín J. Predictors of ineffective cough during a chest infection in patients with stable amyotrophic lateral sclerosis. Am J Respir Crit Care Med 2007;175(12):1266-1271.

17. Janssens JP, Borel JC, Pépin JL, SomnoNIV Group. Nocturnal monitoring of home non-invasive ventilation: the contribution of simple tools such as pulse oximetry, capnography, built-in ventilator software and autonomic markers of sleep fragmentation. Thorax 2011; 66(5):438-445.

18. Volanti P, Cibella F, Sarvà M, De Cicco D, Spanevello A, Mora G, La Bella V. Predictors of non-invasive ventilation tolerance in amyotrophic lateral sclerosis. J Neurol Sci 2011;303(1-2):114-118.

19. Windisch W, Storre JH, Sorichter S, Virchow JC Jr. Comparison of volume and pressure limited NPPV at night: a prospective randomized cross-over trial. Respir Med 2005;99(1):52-59.

20. Schönhofer B, Sonneborn M, Haidl P, Böhrer H, Köhler D. Comparison of two different modes for non-invasive ventilation in chronic respiratory failure: volume versus pressure controlled device. Eur Respir J 1997;10(1):184-191.

21. Tuggey JM, Elliott MW. Randomized cross-over study of pressure and volume non-invasive ventilation in chest wall deformity. Thorax 2005;60(10):859-864.

22. Kleopa KA, Sherman M, Neal B, Romano GJ, Heiman-Patterson T. Bipap improves survival and rate of pulmonary function decline in patients with ALS. J Neurol Sci 1999;164(1):82-88.

23. Atkeson AD, RoyChoudhury A, Harrington-Moroney G, Shah B, Mitsumoto H, Basner RC. Patient-ventilator asynchrony with nocturnal noninvasive ventilation in ALS. Neurology 2011;77(6):549555 .

24. Crescimanno G, Canino M, Marrone O. Asynchronies and sleep disruption in neuromuscular patients under home noninvasive ventilation. Respir Med 2012;106(10):1478-1485.

25. Fanfulla F, Delmastro M, Berardinelli A, Lupo ND, Nava S. Effects of different ventilator settings on sleep and inspiratory effort in patients with neuromuscular disease. Am J Respir Crit Care Med 2005; 172(5):619-624.

26. Farrero E, Prats E, Povedano M, Martínez-Matos JA, Manresa F, Escarrabill J. Survival in amyotrophic lateral sclerosis with home mechanical ventilation. The impact of systematic respiratory assessment and bulbar involvement. Chest 2005;127(6):2132-2138.

27. Jackson CE, Lovitt S, Gowda N, Anderson F, Miller RG. Factors correlated with NPPV in ALS. Amyotroph Lateral Scler 2006;7(2): 80-85.

28. Bach JR, Bianchi C, Aufiero E. Oximetry and indications for tracheotomy for amyotrophic lateral sclerosis. Chest 2004;126(5):15021507.

29. Sancho J, Servera E, Bañuls P, Marin J. Predictors of need for noninvasive ventilation during respiratory tract infections in medically stable non-ventilated subjects with amyotrophic lateral sclerosis. Respir Care 2015;60(4):492-497.

30. Servera E, Sancho J. Non-invasive ventilation in amyotrophic lateral sclerosis. Lancet Neurol 2006;5(4):291-292. 